\title{
Visibility of Wearable Sensors as measured using Eye Tracking Glasses
}

\author{
Meggan King ${ }^{1}$, Feiyan $\mathrm{Hu}^{2}$, Joanna McHugh ${ }^{3}$, Emma Murphy ${ }^{1}$, Eamonn Newman ${ }^{2}$, \\ Kate Irving $^{1}$, Alan F. Smeaton ${ }^{2}$ \\ ${ }^{1}$ School of Nursing \& Human Sciences, Dublin City University \\ 2 INSIGHT: Centre for Data Analytics and School of Computing, Dublin City University \\ ${ }^{3}$ Institute of Neuroscience, Trinity College Dublin \\ \{meggan.king25, feiyan.hu4\}@mail.dcu.ie, mchughje@tcd.ie, \\ \{emma.murphy, eamonn.newman, kate.irving, alan.smeaton\}@dcu.ie
}

\begin{abstract}
Sensor technologies can enable independent living for people with dementia by monitoring their behaviour and identifying points where support may be required. Wearable sensors can provide such support but may constitute a source of stigma for the user if they are perceived as visible and therefore obtrusive. This paper presents an empirical investigation exploring the extent to which wearable sensors are perceived as visible. 23 Participants wore eye tracking glasses, which superimposed the location of their gaze onto video data of their panorama. Participants were led to believe that the research entailed a subjective evaluation of the eye tracking glasses. A researcher wore one of two wearable sensors during the evaluation enabling us to measure the extent to which participants fixated on the sensor during a one-on-one meeting. Results are presented on the general visibility and potential fixations on two wearable sensors, a wrist-work actigraph and a lifelogging camera, during normal conversation between two people.
\end{abstract}

Keywords: Eye-tracking Glasses, Wearable Sensors, Assistive Technology, Dementia, Fixations

\section{$1 \quad$ Introduction}

Assistive technology has great potential to support the functional requirements of people with dementia and enable independent living, delaying and perhaps eradicating the need for institutionalisation. Ambient assistive living and sensor technologies can help to keep people living independently in the home [1], by monitoring their behaviour and identifying points where support may be required $[2,3,4]$. A central difficulty in living with dementia is the associated stigma [5]. An important part of dementia care research, then, is to reduce the sources and impact of this stigma. However, assistive technologies may themselves constitute a source of stigma if they are considered obtrusive or even visible by the person with dementia or their caregiver [6,7]. This obtrusiveness is particularly relevant when exploring the potential of wearable 
sensors to enhance independent living for people with dementia. One of the fundamental principles of responsible technology design for dementia is ensuring that sensors are not obtrusive in any way [8]. Hensel et al. [7] have defined obtrusiveness in this context as "characteristics or effects associated with the technology that are perceived as undesirable and physically and/or psychologically prominent". Therefore the visibility of wearable sensors may constitute obtrusiveness. In this study we investigate the extent to which relevant wearable sensors are perceived within dyadic interactions. Using eye-tracking technologies we can quantify the visual attention given to these sensors in a controlled experimental situation, and extrapolate about the visibility of these sensors. It is intended that the results from this experiment will inform the design and choice of sensors used to support people with dementia, by minimising obtrusiveness.

\section{Wearable Sensors and the Dem@Care Toolbox Approach}

The Dem@Care ${ }^{1}$ project aims to develop a technological support and monitoring system for people with dementia. While the Dem@Care project also involves work in diagnostic laboratories and nursing homes, our focus is on the private home-based deployment of the Dem@Care system. This system uses sensor technologies and feedback to enable the individual to remain independently at home, and optimise wellbeing. The Dem@Care system stems from a person-centred, user-led philosophy [9]. We have defined a "toolbox" approach to the deployment of sensor technology for people with dementia. This approach constitutes a personalised system, built by the person with dementia from 'tools' and software components made available by the researchers, thus constituting an empowering, person-centred approach to care [9].

Part of the Dem@Care toolbox approach involves "wearable" sensors, which are fixed to the body or clothing of the individual. These include the SenseCam (Figure 1a), a camera which hangs by a lanyard around the neck, and the Philips DTI-2 sensor, an actigraphy device with accelerometer and galvanic skin response measures, which is worn as a wristwatch. We were particularly interested in evaluating these sensors in this present visibility investigation so that the results could directly inform our lead users in this project. As the Philips device (a research prototype) was not available at the time of testing, we have chosen to test a similar actigraphy device, the LARK wrist sensor (Figure 1b).

\footnotetext{
${ }^{1}$ http://www.demcare.eu
} 


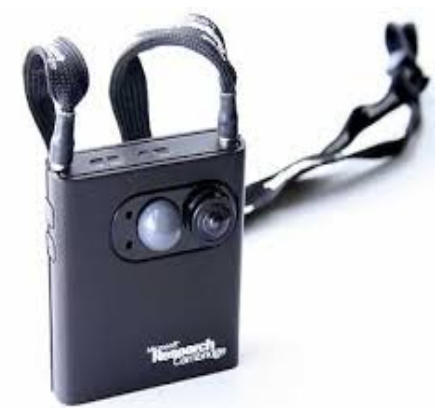

Fig. 1a. SenseCam

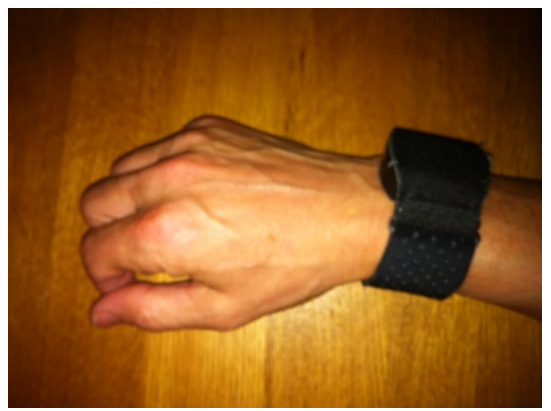

Fig. 1b. LARK Sensor worn on wrist

\section{$3 \quad$ Visibility Experiment}

We investigate, as our primary hypothesis, whether the sensors worn by the researcher are visible as defined by frequency of fixations made during dyadic interaction, as collected via eye tracker recordings made by the participant. In order to explore the visibility of both the neck-based SenseCam and the wrist-worn LARK sensor, we divided participants into two groups to test both sensors (10 participants in each condition). To explore general areas of fixation without a sensor we also ran a small control condition with no sensor (3 participants).

All participants wore Tobii eye tracking glasses (Figure 2a), which tracked the focus point of a subject's gaze and superimposed this onto video data of their panorama. The researcher comprised part of this panorama, while wearing one of the above sensors (either SenseCam or the LARK wrist sensor, or no sensor for control group), and analytics from the recorded gaze allowed us to investigate the extent to which the sensor is the subject of visual attention, if at all. The placement of infra-red (IR) markers on the sofa around the researcher (acting as location anchors) was crucial to accurately aggregate quantitative fixation data. All IR markers were placed in the same two-dimensional plane as the sensors to enhance accuracy, because the video does not contain depth information. We attached 6 IR makers to the sofa where the researcher sat for every evaluation session (see Figure $2 b$ ).

\subsection{Participants}

23 participants were recruited by email for this study from the student and staff population at Dublin City University. Researchers or students specialising in the area of sensor research were precluded from participating. Reported history of psychiatric disorders with a social dysfunctional component (schizophrenia, some personality disorders including autistic spectral disorder) precluded participation, since in many of these disorders, fixation upon the face of a stranger is impaired. Participants wearing glasses were also precluded from taking part in the study, as it is difficult to calibrate and use the eye tracking glasses over another pair of glasses. 14 males and 9 
females, all with normal or corrected-to-normal (with contact lenses) vision, volunteered to participate in the study, between the ages of 19 and $46(\mathrm{M}=28, \mathrm{SD}=8)$.

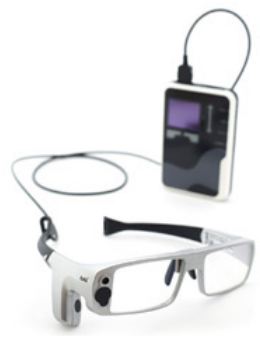

Fig. 2a. Tobii Eye tracking glasses

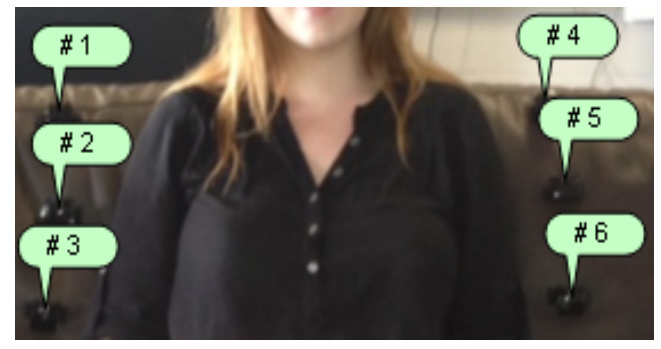

Fig.2b. Placement of IR markers on sofa behind researcher

\subsection{Protocol}

At the time of recruitment and during the experiment, participants were told that the research was investigating the comfort and potential applications of the eyetracking glasses. Before the participant was greeted, the researcher affixed the sensor to her person, in order to maintain visual environmental consistency throughout the experiment's duration. Path of entry to the experimental room, visual distractions in the room, seating arrangements, orientation, and researcher's appearance and conversation were all kept consistent throughout for all 23 participants. Following informed consent, participants were asked to complete a short visual task in order to calibrate the glasses. This involved standing 1 metre away from a yellow IR marker on a wall, and following this marker with their gaze as the researcher moved it around the wall. The researcher then invited participants to sit opposite her on a sofa. The researcher sat on the sofa opposite, with IR markers fixed around her, and proceeded to describe the Tobii glasses. The researcher then asked the participant a number of questions regarding the comfort of the glasses, how they found the calibration process and a number of open ended questions on potential benefits and uses for the glasses. The researcher engaged with the subject at all times during this period, during which the social norm would be not to stare at clothes, jewellery or anything out of the ordinary worn by the researcher, though when not being engaged in eye-to-eye contact, quick glances at something unusual would also constitute normal behaviour. Since the eye tracked sampled gaze at $25 \mathrm{~Hz}$, these quick glances would be measurable. Following this, participants were thanked and told that they would soon receive a debriefing email. In the debriefing e-mail participants were informed of the true aims of the study and asked a series of questions to determine the noticeability, and extent thereof, of the sensors worn. 


\section{$4 \quad$ Results}

Data generated from heat map analysis revealed that the majority of participants for both sensor conditions and control group, fixated on the researchers face/head for the largest proportion of the evaluation time (figure 3 illustrates heat map data for each condition). The average time that participants spent in conversation with the researcher and having their gaze recorded was 256 seconds $(S D=64 \mathrm{~s})$. Participants spent an average of $73 \%$ total time fixating on the researcher's face, in comparison to less than an average of $1 \%$ of the total time fixated on sensors. When we analysed the heat maps individually, 20 participants fixated primarily on the researcher's face/head and 3 participants fixated on other areas. Of these 3, one fixated on the researcher's shoulder, one on her neck and one participant fixated on their own reflection in a glass panel behind the researcher. Data generated from the heat map analysis also revealed that participants spent approximately $1 \%$ of the overall time fixating on the researcher's hands. The average percentage of fixation time recorded on the head/face for the SenseCam condition was $74.24 \%(\mathrm{SD}=16.14 \%)$. For the LARK condition, participants fixated on the head/face $71.98 \%(\mathrm{SD}=27.60 \%)$ of the total evaluation time. The average proportion of fixation time recorded on each sensor was $0.78 \%$ for the SenseCam $(\mathrm{SD}=1.12 \%)$ and $0.37 \%$ for the LARK $(\mathrm{SD}=0.79 \%)$.

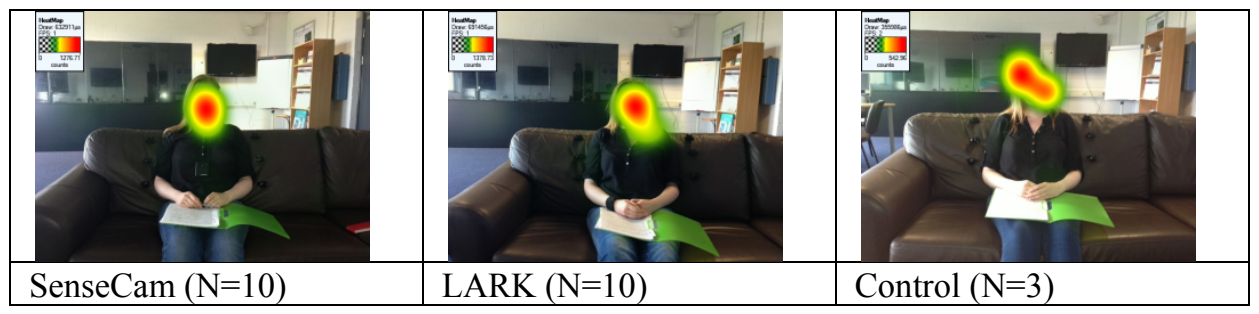

Fig. 3. Heat map data for each condition

\subsection{Detailed Video Analysis}

To investigate in more detail the actual frequency of fixations on sensors and the researcher's hands, we conducted a fine-grained analysis of fixations on both sensors and hands in all conditions by manually analysing each participant video. Each video was played in slow motion and was paused at every point of fixation on the sensor or the hands and the time of fixation was recorded. This was repeated to reduce the chance of error. For the SenseCam condition, fixations were recorded on the SenseCam and the hands (as one score). For the LARK condition, fixations were recorded on the LARK and on the hands (as three scores: one for the hands, one for the left hand and one for the right hand). The analyst also recorded relevant comments or particulars in fixation data that were made during the videos.

Data were frequency of fixations on the sensors, as well as fixations on a secondary point in the visual field (hands). The manipulated variable was sensor type with 
two levels (SenseCam or LARK). Data were screened for outliers and assessed for normality of distribution. There were no outliers and both the kurtosis and skewness test indicated no serious departures from normality (all coefficients resulted in absolute values of less than 1). Levene's test for homogeneity of group variance was also non-significant. The Shapiro-Wilk test was conducted to test for normality, due to having a small sample size, and found to be non-significant, indicating normality of distribution [SenseCam: $\mathrm{D}_{10}=1.90, \mathrm{p}<0.05$; LARK, $\mathrm{D}_{10}=2.54, \mathrm{p}<0.05$ ].

Number of fixations on each sensor was recorded for every participant. A mean of 5 fixations for the LARK ( $\mathrm{SD}=4.62$ ) and of 2.87 fixations for the SenseCam $(\mathrm{SD}=$ 2.89) were recorded, and an independent samples t-test was then conducted, which found that there was no significant difference between fixations on the two sensor types $\left(\mathrm{t}_{18}<1\right)$.

A 2 x 2 ANOVA was used to investigate potential effects of gender and sensor type on frequency of fixations (see Table 1). There was no statistically significant interaction effect $\left(\mathrm{F}_{1,18}<1\right)$, nor was there a main effect for gender $\left(\mathrm{F}_{1,18}<1\right)$, nor sensor type

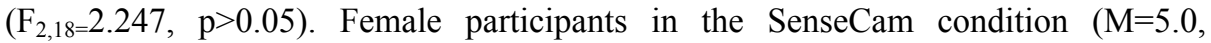
$\mathrm{SD}=2.65$ ) had a higher mean frequency of fixations than the males in the SenseCam condition $(\mathrm{M}=3.14, \mathrm{SD}=3.18), \mathrm{d}=0.6$ (See Figure $4 \mathrm{a})$.

\begin{tabular}{lllll}
\hline Condition & Gender & Mean & Std. Deviation & $\mathbf{N}$ \\
\hline \multirow{2}{*}{ SenseCam } & Male & 3.1429 & 3.18479 & 7 \\
& Female & 5 & 2.64575 & 3 \\
& Total & 3.7 & 3.0203 & 10 \\
\hline \multirow{2}{*}{ LARK } & Male & 3 & 2.88675 & 7 \\
& Female & 2.6667 & 3.05505 & 3 \\
& Total & 2.9 & 2.76687 & 10 \\
& Female & 0 & 0 & 3 \\
\multirow{2}{*}{ Control } & Total & 0 & 0 & 3 \\
& Male & 3.0714 & 2.92112 & 14 \\
& Female & 2.5556 & 2.96273 & 9 \\
& Total & 2.8696 & 2.88104 & 23 \\
\hline
\end{tabular}

Table 1 Means and standard deviations for sensor fixation scores.

Independent t-tests were conducted to determine whether or not participants fixate on the hands in a similar way across each of the three sensor type conditions (SenseCam, LARK and control). Hands were fixated on with lower frequency when the SenseCam was worn, $(M=4.2, S D=3.29)$, than when the LARK was worn $(M=7.3$, $\mathrm{SD}=5.14)$. However, no significant difference was found $\left(\mathrm{t}_{18}=-1.6, \mathrm{p}>0.05, \mathrm{~d}=0.7\right)$. Hands were fixated upon more frequently when SenseCam was worn $(\mathrm{M}=4.2$, $\mathrm{SD}=3.29)$ than in the control condition when no sensor was worn $(\mathrm{M}=0, \mathrm{SD}=0)$ and this difference was not significant $\left(\mathrm{t}_{11}=2.142, \mathrm{p}>0.05\right)$. In the LARK condition 
$(M=7.3, S D=5.14)$, participants fixated significantly more often on the hands than in the control condition $(\mathrm{M}=0, \mathrm{SD}=0),\left(\mathrm{t}_{11}=2.384, \mathrm{p}>0.05\right)$. Frequency of fixations on the hands of the researcher are illustrated in Figure $4 \mathrm{~b}$.

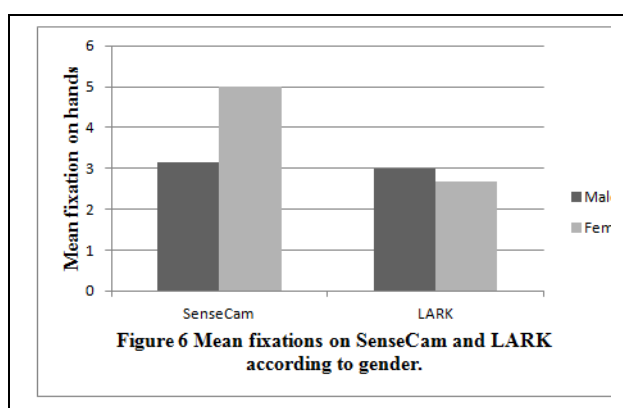

Fig. 4a. Mean fixations on SenseCam and LARK according to gender.

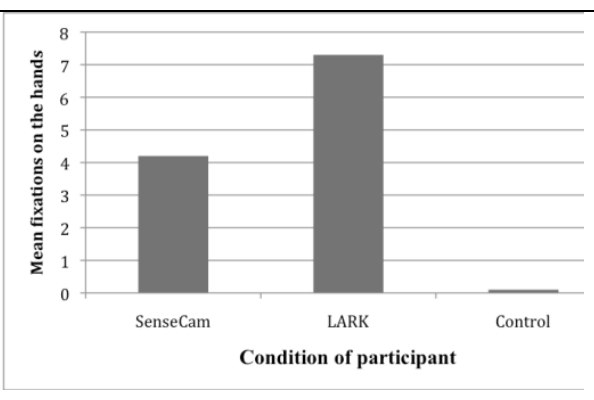

Fig. 4b. Mean fixations on hands for each sensor condition

To explore the possibility that the participant might fixate on one hand more than the other, independently of presence of the wrist-worn LARK (worn on the right wrist), the frequency of fixations on each hand was also recorded for the control group in which no sensors were worn. An independent samples t-test was conducted to investigate if participants fixated on each hand in a similar way, regardless of the researcher wearing the LARK. Frequency of fixations on the left hand were compared between the LARK condition $(\mathrm{M}=4.3, \mathrm{SD}=3.16)$ and the control condition (Mean $=3, \mathrm{SD}=$ $2.65)$, and no significant difference was found $\left(\mathrm{t}_{11}=0.642, \mathrm{p}>0.05\right)$. Frequency of fixations on the right hand were also compared between the LARK condition $(\mathrm{M}=3$, SD $=2.54)$ and the control condition $(\mathrm{M}=2.67, \mathrm{SD}=2.89)$, and again no significant difference was found $\left(\mathrm{t}_{11}<1\right)$.

\subsection{Post Study Questionnaires}

None of the 12 respondents to the post-study questionnaire reported seeing the sensor as worn by the researcher. 9 participants ranked the sensor as not noticeable at all. 1 participant in the wrist sensor condition reported seeing an ID badge, 1 participant mistook the IR markers for sensors and 1 participant reported that she perceived the glasses (spectacles) worn by one researcher as potentially containing a sensor. All participants responded yes when asked if they would wear one of the wearable sensors if they thought it would be of some benefit to their life. Table 2 highlights that, even though potential fixations were identified in the manual video analysis for many of the participants, they did not report noticing a sensor after the study. 


\begin{tabular}{llll}
\hline Sensor & Gender & Noticed Sensor & Fixations on sensor \\
\hline SenseCam & $\mathrm{M}$ & $\mathrm{N}$ & 0 fixations \\
SenseCam & $\mathrm{M}$ & $\mathrm{N}$ & 0 fixations \\
SenseCam & $\mathrm{F}$ & $\mathrm{N}$ & 4 fixations \\
SenseCam & $\mathrm{M}$ & $\mathrm{N}$ & 0 fixations \\
SenseCam & $\mathrm{M}$ & $\mathrm{N}$ & 6 fixations \\
SenseCam & $\mathrm{M}$ & $\mathrm{N}$ & 3 fixations \\
\hline LARK & $\mathrm{M}$ & $\mathrm{N}$ & 1 fixation \\
LARK & $\mathrm{F}$ & $\mathrm{N}$ & 2 fixations \\
LARK & $\mathrm{M}$ & $\mathrm{N}$ & 5 fixations \\
LARK & $\mathrm{M}$ & $\mathrm{N}$ & 0 fixations \\
LARK & $\mathrm{F}$ & $\mathrm{N}$ & 6 fixations \\
\hline Control & $\mathrm{M}$ & $\mathrm{N}$ & N/A \\
\hline
\end{tabular}

Table 2 Participants who reported noticing sensor vs. actual fixations on sensor

\section{Discussion}

This investigation concerned the fixation of participants' gaze upon sensors worn by the researcher, in two conditions. Our primary aim was to ascertain whether the sensors were fixated upon at a rate significantly higher than any other area, and we conclude that they were not. The sensors do not appear to constitute a particularly visible or obtrusive item even in direct face-to-face conversations, at least with reference to the number of fixations made upon them. Further, neither type of sensor was differentially more or less visible than the other, indicating that both sensor types explored in the current analysis can be said to be non-obtrusive as measured by eye tracker fixation metrics. The sensors are worn on the wrist and around the neck on a lanyard respectively, potentially drawing attention to two different body parts. It was suspected that gender may impact area of fixation (the researcher was female). As such we investigated frequency of fixations on the sensors as a function of gender and no such relationship was found; it appears that male and female participants fixated equally upon the two sensor types. Thus the data remained pooled for the duration of the analyses.

We also investigated whether the wrist-worn sensor attracted more fixations to the hands, but there was no difference across sensor conditions in the frequency of fixations to the hands. Nor was there a difference found in fixations to the left or the right hand, indicating that the LARK sensor (worn on the right hand throughout) did not attract increased levels of fixation. The hands were nevertheless a region of significant fixation across participants, which may reflect the adaptive importance of perception of hand-related action from others. This area is responsible for the majority of instrumental actions carried out by others and is therefore an important area to accurately and sufficiently monitor [10].

There are limitations to the heat map analyses performed. While this data shows overwhelmingly that participants spent a relatively small proportion of the time fixating on both wearable sensors in comparison to the head area, the snapshots used to 
generate heat maps are only assumed to be representative of the eye tracking video rather than being totally accurate, as the area that we are investigating moves relative to the IR markers. In this case, it is likely that the researcher moved her head or torso or used gestural language. The more the researcher moves, the less accurate the fixation time. As we cannot presently quantify the movements of researcher and resultant artefact in the video data, further analyses are necessary to accurately determine actual fixations on the sensors, as well as to detect quick glances.

There are a number of limitations to report in the current study, namely, sampling and environmental issues. The current study included a limited sample size, a restricted pool of participants and was not gender-balanced which means that the sample does not constitute generalisable data and could result in reduced power. Furthermore, environmental background noise was notable in the current study. The presence of infrared (IR) markers attached to the seat surrounding the researcher during the study, may have been a distraction, as several participants remarked on the IR markers after the study, fixated on them during the study or mistakenly reported them as the sensors in the follow-up questionnaire. Also, due to changes in natural daylight, reflections off the glass panelling behind the interviewer varied across participants, with one participant primarily fixating on their reflection throughout the study. These limitations related to the experimental set up are important issues to highlight for future experiments using the eye tracking glasses to explore a physical environment. Furthermore, the issues identified in the results section in relation to the accuracy of the automated heat map data analysis are also a worthwhile area of further investigation.

\section{Conclusion}

In spite of the limitations identified above, the data produced in this experiment overwhelming illustrates that participants spent a very small proportion of the evaluation time fixating on wearable sensors, in comparison to lengthy fixations on the researcher's face or other areas of the room. While the more detailed annotated video analysis revealed that some participants fixated a number of times in the areas of both wearable sensors, none of the participants reported having noticed the sensors in post study questionnaires. This is a positive result to report in the context of promoting wearable sensors to enable independent living for people with dementia. Wearable sensors can provide such support without constituting an additional source of stigma for the user. This study has revealed that while observers did minimally fixate on the two wearable sensors evaluated in this experiment, sensors were not consciously noticed by observers and therefore can be considered unobtrusive.

\section{Acknowledgements}

This research is supported by the European Community 7th Framework Programme (FP7/2007- 2013) under grant agreement 288199 (Dem@Care) and by Sci- 
ence Foundation Ireland under grant SFI/12/RC/2289 (INSIGHT). We would also like to thank all participants for their time and effort in taking part in this study.

\section{References}

1. Drennan J, Treacy MP, Butler M, Byrne A, Fealy G, Frazer K, Irving K.: Support networks of older people living in the community International Journal of Older People Nursing 3 (4), 234-242 (2008)

2. Hoof, J van, Kort H, Rutten P, and Duijnstee M.: Ageing-in-place with the use of ambient intelligence technology: Perspectives of older users. International Journal of Medical Informatics;80:310-331 (2011)

3. Orpwood, R., Gibbs, C., Adlam, T., Faulkner, R. \& Meegahawatte, D. The design of smart homes for people with dementia: user-interface aspects. Universal Access Information Society 4: 156-164 (2005)

4. Biswas, J., Tolstikov, A., Jayachandran, M., Foo, V., Wai, A.A.P., Phua, C., Huang, W., Shue, L., Gopalakrishnan, K., Lee, J. and Yap, P.: Health and wellness monitoring through wearable and ambient sensors: exemplars from home-based care of elderly with mild dementia. Annals of Telecommunications - Annales Des Télécommunications, 65(9-10), pp.505 - 521.(2010)

5. Batsch, N.L., Mittelman, M.S. \& Alzheimer's Disease International: World Alzheimer Report 2012: Overcoming the stigma of dementia. London, UK: Alzheimer's Disease International. (2012).

6. Demiris, G., Hensel, B.: "Smart homes" for patients at the end of life, Journal of Housing for the Elderly 23 (1) 106-115 (2009)

7. Hensel, B. K., Demiris, G. and Courtney, K. L. Defining obtrusiveness in home telehealth technologies: A conceptual framework. Journal of the American Medical Informatics Association, 13: 428-431 (2006).

8. Orpwood, R., Faulkner, R., Gibbs, C. \& Adlam, T. A design methodology for assistive technology for people with dementia. In GM Craddock, LP McCormack, RB Reilly and HTP Knopps (Eds). Assistive technology: Shaping the future. (2003)

9. McHugh, J.E., Smeaton, A.F., Irving, K. \& Newman, E.: The Dem@Care Toolbox Approach. Position paper presented at the SIGCHI 2013 Workshop on Designing for and with Vulnerable People, CHI April 2013, Paris, France (2013).

10. Grezes, J., Costes, N., and Decety, J., Top down effect of the strategy on the perception of human biological motion: a PET investigation, Cognitive Neuropsychology, 15, 553-582 (1998). 